Original

\title{
Absentismo laboral por causa médica en trabajadores del área operativa de una compañía de extracción de minerales en Colombia, 2011
}

\author{
Sickness absence in workers from an operating area of a mining company in \\ Colombia, 2011
}

\section{Elsa María Vásquez Trespalacios}

\section{Grupo de Investigación en Epidemiología y Bioestadística. Facultad de Medicina. Universidad CES. Medellín (Colombia)}

\section{Recibido: 21-08-12}

Aceptado: 20-12-12

\section{Correspondencia \\ Elsa María Vásquez Trespalacios \\ Facultad de Medicina. Universidad CES \\ Calle 10 A \# 22-04, Medellín (Colombia). \\ Teléfono: 57-4-4440555 \\ Correo electrónico: evasquez@ces.edu.co}

Resumen

Introducción: El sector minero es uno de los más dinámicos de la economía en Colombia, sin embargo, poco se conoce acerca del ausentismo laboral que enfrenta.

Objetivo: Describir la magnitud del ausentismo laboral por causa médica en el área operativa de una empresa minera Colombiana, durante el año 2011.

Material y métodos: estudio descriptivo, transversal, en el que se analizaron 130 ausencias por causa médica. Los episodios se categorizaron según grupos diagnósticos de la Clasificación Internacional de Enfermedades versión 10 (CIE-10) y se calcularon indicadores de ausentismo.

Resultados y discusión: $66.9 \%$ de las ausencias se debió a enfermedad general y el 33.3\% a accidentes de trabajo. El promedio de ausencia es de $4.28+/-5.64$ días por trabajador. Las enfermedades musculoesqueléticas y los traumatismos y envenenamientos tuvieron los mayores días de ausencia, similar a lo reportado por otros investigadores. Los accidentes de trabajo generaron 315 días de ausencia. 19 ausentistas repetitivos aportaron en total 100 días perdidos. La edad promedio de los ausentistas repetitivos es de 28.6 años+/-6.7. Los ausentistas crónicos generaron una pérdida de 258 días por cada 100 trabajadores programados.

Med Segur Trab (Internet) 2013; 59 (230) 93-101

Palabras Clave: Ausentismo laboral, dias de trabajo perdido, incapacidad, enfermedades, minería.

Abstract

Introduction: The mining sector is one of the most dynamic in Colombian economy, however, little is known about absenteeism. Aim: To describe the extent of sickness absence in an operating area of a mining company in Colombia, in 2011. 
Material and Metbods: A descriptive, cross-sectional study, which analyzed 130 sickness absences. The episodes were categorized according to diagnostic groups of the International Classification of Diseases version 10 (ICD-10) and calculated rates of absenteeism.

Results and discussion: $66.9 \%$ of absences were due to general illness and 33.3\% of accidents at work. The average absence is $4.28+/-5.64$ days per worker. Most of absence days were generated by musculoskeletal diseases and injuries and poisoning, similar to that reported by others. Work accidents generated 315 days of absence. 19 repetitive absentee contributed a total of 100 lost days. The average age of a repetitive absentee is 28.6 years $+/-6.7$. The chronic absentee generated a loss of 258 days per 100 scheduled workers.

Med Segur Trab (Internet) 2013; 59 (230) 93-101

Key Words: Absenteeism, mining, sickness absence, diagnostic group. 


\section{INTRODUCCIÓN}

Según la Organización Internacional del Trabajo, se entiende por ausentismo: "La no asistencia al trabajo por parte de un trabajador del que se pensaba que iba a asistir, quedando excluidos los períodos vacacionales y las huelgas". Y el ausentismo laboral de causa médica, como el período de baja laboral atribuible a una incapacidad del individuo, excepción hecha para la derivada del embarazo normal o prisión"1.

Existen diferentes modelos teóricos que explican el ausentismo laboral:

Los modelos de evitación laboral; que suponen que el descontento en el trabajo es la primera causa de ausencia de un trabajador y por lo tanto esta ausencia es una forma de escape o huida a condiciones calificadas negativamente por parte del trabajador. Durante mucho tiempo las investigaciones realizadas en este tema fueron guiadas bajo los supuestos de este modelo².

Sin embargo, la existencia de la asociación entre ausentismo y satisfacción laboral no es concluyente ${ }^{3}$ existen otros factores que pueden explicar el fenómeno del ausentismo con mayor exactitud ${ }^{4,5}$.

Otros modelos teóricos se inclinan hacia la concepción de que las ausencias laborales son un resultado de la adaptación que tiene el trabajador a su medio laboral ${ }^{4}$.

Los modelos de decisión que se ubican desde una perspectiva individual en dónde el trabajador se ausenta de su lugar de trabajo en un proceso racional de sopesar los riesgos y beneficios de su ausencia, de manera tal que cuando un empleado no asiste a su lugar de trabajo es debido a que ha realizado una valoración de las posibles consecuencias que su inasistencia podría ocasionar ${ }^{6,7}$.

Otros modelos incorporan propuestas que intentan explicar el ausentismo laboral a través de las características de personalidad de los individuos, en los últimos años ha crecido el cuerpo de literatura que aborda los factores personales como variables explicativas de las ausencias de los trabajadores ${ }^{5,8-10}$.

A partir de los modelos anteriores, Steers y Rhodes proponen un modelo integrado para explicar el comportamiento ausentista. Este modelo comprende tres grupos de factores que afectan la asistencia al lugar de trabajo; la cultura de ausencia en la organización, las políticas y prácticas organizacionales y las actitudes, valores y metas del empleado ${ }^{11}$.

\section{Ausentismo en la empresa minera}

Mesa y Kempfer en 2004 realizan un análisis del ausentismo laboral en Chile recogiendo datos de varias investigaciones al respecto, según el tipo de empresa, concluyendo que para la empresa minera la tasa de ausentismo fue de 11.2 día anuales por trabajador y encontrando en orden de importancia como causa de ausencia médica las enfermedades respiratorias, osteomusculares, digestivas y traumatismos ${ }^{12}$.

Cantaurias et al. analizaron el ausentismo por causa médica y no médica en trabajadores de sexo masculino que realizaban turnos rotativos en una compañía de extracción de minerales, reportando que el índice de ausentismo general era de 8.8 días de ausencia por 100 días de trabajo, de estas ausencias, el 85\% estaba representado por causa médica. Entre las enfermedades analizadas, las del tracto respiratorio fueron las más prevalentes, sin embargo, los traumatismos y las enfermedades osteomusculares tenían la mayor severidad, con valores por encima de los 10 días perdidos ${ }^{13}$.

En los últimos años, el sector de extracción de minerales en se ha consolidado como uno de los más dinámicos en la economía de Colombia, esto se puede evidenciar en el comportamiento de su Producto Interno Bruto, que en el último año registró un crecimiento del 5.9\%, el más elevado desde el año $2007^{14}$. A pesar de la importancia del sector de extracción de minerales en Colombia, no se tienen cifras consolidadas del ausentismo laboral que se presenta en este sector de la economía. 
El objetivo del presente estudio fue estimar la magnitud del ausentismo laboral en una empresa de extracción de minerales en Colombia y hacer un acercamiento a las posibles causas del fenómeno.

\section{MATERIAL Y MÉTODOS}

Se realizó un estudio con enfoque cuantitativo, de tipo descriptivo para estimar la magnitud del ausentismo laboral en el área operativa de una empresa de extracción de minerales en Colombia y poder realizar un acercamiento a las causas de este fenómeno.

Se evaluaron todos los registros de ausencias medicamente certificadas, presentadas en el periodo de tiempo comprendido entre el $1 .^{\circ}$ de enero de 2011 y el 31 de diciembre de 2011, se registraron algunos datos del trabajador ausente. En total se analizaron 130 ausencias. El motivo principal de la ausencia se categorizó por sistemas de acuerdo con los diagnósticos de la Clasificación Internacional de Enfermedades de la Organización Mundial de la Salud (CIE-10) ${ }^{15}$ con el fin de encontrar tendencias en el comportamiento del ausentismo. Con respecto al tiempo, se registró el mes del año y el día de la semana en el que se presentó el episodio de ausencia.

Las ausencias debidas a licencia por maternidad se excluyeron del estudio al no estar clasificadas por causa médica sino legal.

Se calcularon los índices de frecuencia, severidad, porcentaje de tiempo perdido, duración promedio del episodio de ausencia, duración promedio de días perdidos por persona ausente, de acuerdo con lo establecido en la Norma Técnica Colombiana NTC $3793^{16}$.

Índice de frecuencia IF: Es la proporción de episodios de ausencia por cada 100 trabajadores programados de tiempo completo durante el período

$$
\mathbf{I F}=\frac{\mathrm{N}^{\circ} \text { de episodios de ausencia en el período }}{\mathrm{N}^{\circ} \text { total de horas-hombre programadas en el período }^{\circ}} \times 240.000
$$

La constante de 240.000 resulta, de acuerdo con la legislación colombiana, de multiplicar:

100 (trabajadores) x 48 (horas laboradas en la semana) x 50 (semanas del año). A las 52 semanas del año se descuentan 2 semanas correspondientes al periodo de vacaciones.

Horas-hombre programadas $=\mathrm{N} .^{\circ}$ total de trabajadores $\mathrm{x} 50$ semanas $\mathrm{x} 42$ horas

Índice de severidad IS: Es el número de días perdidos por cada 100 trabajadores programados de tiempo completo durante el período.

$$
\text { IS }=\frac{\mathrm{N} .^{o} \text { total de días perdidos en el período }}{\mathrm{N}^{\mathrm{o}} \text { total de horas-hombre programadas en el período }^{\circ}} \times 240.000
$$

Porcentaje de tiempo perdido PTP

$$
\mathbf{P T P}=\frac{\text { Total de días perdidos en el período } \times 8}{\text { N. }^{\circ} \text { total de horas-hombre programadas en el período }} \times 100
$$

Duración promedio del episodio de ausencia DPE:

$$
\text { DPE }=\frac{\mathrm{N} .^{o} \text { total de días perdidos en el período }}{\mathrm{N} .^{\circ} \text { total de episodios de ausencia en el período }}
$$


Duración promedio de días perdidos por persona ausente DPD:

$$
\mathbf{D P D}=\frac{\mathrm{N} .^{\circ} \text { total de días perdidos en el período }}{\mathrm{N} .^{\circ} \text { total de trabajadores ausentes en el período }}
$$

Los datos fueron procesados con el programa estadístico SPSS versión 18.

\section{RESULTADOS}

En el periodo comprendido entre el $1 .^{\circ}$ de enero de 2011 y el 31 de diciembre de 2011 se presentaron 130 episodios de ausencias, que generaron 673 días perdidos. El $\mathbf{9 4 . 6 1 \% ~ d e ~ l a s ~ a u s e n c i a s ~ s e ~ g e n e r a r o n ~ p o r ~ t r a b a j a d o r e s ~ d e ~ s e x o ~ m a s c u l i n o . ~ D e ~ a c u e r d o ~}$ con el área de trabajo, el $73 \%$ de las ausencias fueron causadas por trabajadores del área de extracción y limpieza del mineral. Según la causa, el $66.9 \%$ de las ausencias se generaron por enfermedad general. El $77 \%$ de las ausencias del periodo se reportaron en trabajadores menores de 34 años.

Tabla I. Distribución del ausentismo por causa médica

\begin{tabular}{ccccc}
\hline & Característica & Frecuencia & Porcentaje & N \\
\hline \multirow{2}{*}{ Sexo } & Femenino & 7 & $5,38 \%$ & 130 \\
& Masculino & 123 & $94,61 \%$ & \\
\hline \multirow{2}{*}{ Área de trabajo } & Extracción y limpieza de mineral & 95 & $73,07 \%$ & \\
& Geología y protección ambiental & 25 & $19,23 \%$ & 130 \\
& Seguridad y mantenimiento & 10 & $7,69 \%$ & \\
\hline \multirow{2}{*}{ Causa de la ausencia } & Enfermedad general & 87 & $66,90 \%$ & 130 \\
& Accidente de trabajo & 43 & $33,07 \%$ & \\
\hline \multirow{2}{*}{ Grupo de edad } & $18-24$ & 51 & $39,23 \%$ & \\
& $25-29$ & 23 & $17,69 \%$ & \\
& $30-34$ & 26 & $20,00 \%$ & \\
& $35-39$ & 12 & $9,23 \%$ & \\
& $40-44$ & 7 & $5,38 \%$ & \\
& $45-49$ & 10 & $7,69 \%$ & \\
& $50-54$ & 1 & $0,77 \%$ &
\end{tabular}

El promedio de ausencia es de $4.28+/-5.64$ días por trabajador ausente. La mayor proporción de ausencias se presentaron los días lunes.

Durante el periodo de estudio se presentaron en total 130 ausencias por causa médica, 43 ausencias (33.6\%) por causa médica se atribuyen a accidentes ocurridos en el lugar de trabajo. El grupo diagnóstico con mayor número de ausencias para el periodo de estudio es aquel que agrupa todas las enfermedades del sistema musculoesquelético. La mayor cantidad de días de ausencia se atribuye a los grupos diagnósticos del sistema musculoesquelético y a los traumatismos y envenenamientos. Los accidentes de trabajo por su parte generaron 315 días de ausencia, explicados en su gran mayoría por la prolongada incapacidad de un trabajador con una fractura de miembro inferior izquierdo, lo cual a su vez hace que el índice de severidad por esta causa sea el mayor entre los demás grupos diagnósticos (195.65 días perdidos por cada 100 trabajadores programados de tiempo completo para el periodo) seguido por las enfermedades del sistema musculoesquelético (117.39). Las mayores duraciones promedio de los episodios de 
ausencia son en su orden; accidente de trabajo (por la razón mencionada anteriormente), enfermedades del sistema osteomuscular y traumatismos y envenenamientos, situación que también se ve reflejada en el índice de días perdidos por persona ausente. No se presentaron ausencias por enfermedades de la piel ni por trastornos del comportamiento y enfermedades mentales.

Tabla II. Índices de ausentismo por causa médica según grupos diagnósticos

\begin{tabular}{|c|c|c|c|c|c|c|c|c|c|}
\hline Grupo diagnóstico & Ausencias & Días & Ausentes & Población & IF & IS & PTP & DPE & DPD \\
\hline $\begin{array}{l}\text { Enfermedades infecciosas y } \\
\text { parasitarias }\end{array}$ & 9 & 12 & 9 & 186 & 5,59 & 7,45 & 0,02 & 1,33 & 1,33 \\
\hline $\begin{array}{l}\text { Enfermedades del sistema } \\
\text { musculoesquelético }\end{array}$ & 27 & 189 & 22 & 186 & 16,77 & 117,39 & 0,39 & 7,00 & 8,59 \\
\hline $\begin{array}{l}\text { Enfermedades del sistema } \\
\text { respiratorio }\end{array}$ & 10 & 17 & 7 & 186 & 6,21 & 10,56 & 0,04 & 1,70 & 2,43 \\
\hline $\begin{array}{l}\text { Traumatismos o } \\
\text { envenenamientos }\end{array}$ & 18 & 96 & 17 & 186 & 11,18 & 59,63 & 0,20 & 5,33 & 5,65 \\
\hline Enfermedades de la piel & 0 & 0 & 0 & 186 & 0,00 & 0,00 & 0,00 & 0,00 & 0,00 \\
\hline $\begin{array}{l}\text { Enfermedades del sistema } \\
\text { genitourinario }\end{array}$ & 2 & 5 & 2 & 186 & 1,24 & 3,11 & 0,01 & 2,50 & 2,50 \\
\hline $\begin{array}{l}\text { Enfermedades del sistema } \\
\text { digestivo }\end{array}$ & 8 & 13 & 8 & 186 & 4,97 & 8,07 & 0,03 & 1,63 & 1,63 \\
\hline $\begin{array}{l}\text { Enfermedades del sistema } \\
\text { nervioso }\end{array}$ & 7 & 16 & 6 & 186 & 4,35 & 9,94 & 0,03 & 2,29 & 2,67 \\
\hline Accidente de Trabajo & 41 & 315 & 32 & 186 & 25,47 & 195,65 & 0,65 & 7,68 & 9,84 \\
\hline TOTAL & 128 & 673 & 109 & 186 & 79,50 & 418,01 & 1,39 & 31,13 & 36,30 \\
\hline
\end{tabular}

Los ausentistas repetitivos son aquellos trabajadores que presentaron eventos repetidos de ausencia de corta duración (inferior a 4 días) en un número superior a la mediana. Para este estudio la mediana de los días de ausencia fue de 2.

Durante el año 2011, 19 trabajadores presentaron 3 o más episodios de ausencia de corta duración, aportando en total 100 días perdidos. La edad promedio de los ausentistas repetitivos es de 28.6 años+/-6.7. El $84.3 \%$ de los ausentistas repetitivos son de sexo masculino y el $64 \%$ pertenecen al área de extracción y limpieza de mineral.

A causa de los ausentistas repetitivos se generan cerca de 37 ausencias por cada 100 trabajadores programados de tiempo completo para el periodo de estudio.

Tabla III. Ausentistas repetitivos

\begin{tabular}{cccrrr}
\hline Causa de la ausencia & Ausencias & Ausentes & Días & \multicolumn{1}{c}{ IF } & \multicolumn{1}{c}{ IS } \\
\hline Enfermedad general & 60 & 17 & 96 & 36,87 & 58,99 \\
Accidente de trabajo & 2 & 2 & 4 & 1,23 & 2,46 \\
\hline TOTAL & $\mathbf{6 2}$ & $\mathbf{1 9}$ & $\mathbf{1 0 0}$ & $\mathbf{3 8 , 1 0}$ & $\mathbf{6 1 , 4 4}$ \\
\hline
\end{tabular}

El término ausentista crónico corresponde al grupo de trabajadores con repetidos episodios de ausencia de larga duración (mayor de 16 días) en un número superior a la mediana

El 100\% de los ausentistas crónicos son de sexo masculino, el promedio de edad es 32.25 años con una desviación estándar de 8.8 años. 
Los ausentistas crónicos generaron una pérdida de 258 días por cada 100 trabajadores programados para el periodo de estudio.

Tabla IV. Ausentistas crónicos

\begin{tabular}{ccccrr}
\hline Causa de la ausencia & Ausencias & Ausentes & Días & \multicolumn{1}{c}{ IF } & \multicolumn{1}{c}{ IS } \\
\hline Enfermedad general & 4 & 3 & 420 & 2,458 & 258,06 \\
Accidente de trabajo & 3 & 1 & 60 & 1,84 & 36,87 \\
\hline TOTAL & $\mathbf{7}$ & $\mathbf{4}$ & $\mathbf{4 8 0}$ & $\mathbf{4 , 3 0}$ & $\mathbf{2 9 4 , 9 3}$ \\
\hline
\end{tabular}

\section{DISCUSIÓN}

Se evaluaron 130 ausencias por causa médica de los trabajadores del área operativa, de las cuales 87 fueron atribuibles a enfermedad general y 43 a accidente de trabajo, encontrándose similitud con lo reportado por otros investigadores ${ }^{13,17,18}$.

De los 186 trabajadores del área operativa, 130 (72\%) se ausentaron por causa médica, superior a lo reportado por Parada et al. en 2005 cuando analizan las ausencias por causa médica del Instituto Autónomo Hospital Universitario de los Andes en Mérida, Venezuela, en donde reporta que el $47 \%$ del total de trabajadores de la institución se ausentaron por esta causa entre el año 2001 y $2003^{19}$.

Las enfermedades con mayor frecuencia son las del sistema musculoesquelético, los traumatismos y envenenamientos y las enfermedades del sistema respiratorio.

El estudio de la cohorte ocupacional prospectiva (GAZEL) en donde se evaluaron todas las ausencias por enfermedad, médicamente certificadas, superiores a los 7 días de duración, reporta que las causas más frecuentes de ausencias médicas en hombres son las enfermedades del sistema musculoesquelético, consistente con lo encontrado en este estudio. Las ausencias generadas por desórdenes mentales tuvieron una prevalencia importante en el estudio GAZEL, en contraposición con lo encontrado en este estudio en donde ninguna de las ausencias evaluadas para el periodo de tiempo entre el $1 .^{\circ}$ de enero y el 31 de diciembre de 2011 se registró por esta causa ${ }^{20}$.

Mesa y Kaempffer evaluando el ausentismo laboral en Chile de acuerdo con el tipo de empresa, concluyen que los estudios analizados para el sector de la minería en conjunto, reportan tasas de ausentismo de $\mathbf{1 1 . 2}$ días anuales por trabajador, superior a lo encontrado en este estudio, en donde el promedio de días de ausencia por trabajador fue de 4.28.

Con relación al diagnóstico de la ausencia, las enfermedades respiratorias fueron las más prevalentes en 3 de 4 artículos evaluados, seguido por las enfermedades del sistema musculoesquelético, el artículo restante muestra mayor prevalencia de las enfermedades musculoesqueléticas seguido de las enfermedades respiratorias ${ }^{12}$.

Gomero y Llap en 2001, analizando el ausentismo laboral en una empresa minera concluyen que las dos principales causas de ausencia por causa médica son la enfermedad común (82.26\%) y los accidentes (16.45\%), con proporciones bastante similares a las encontradas en este estudio. De igual forma, dentro de las causas de ausencia de origen no infeccioso, la mayor proporción se atribuyen a las alteraciones de la columna vertebral y a las patologías del sistema musculoesquelético; $13.59 \%$ y $11.50 \%$, respectivamente ${ }^{21}$.

Los desórdenes musculoesqueléticos y la discapacidad resultante por su causa son comunes en la fuerza laboral alrededor del mundo, representando una gran causa de ausencia por enfermedad que a menudo desemboca en ausencias prolongadas ${ }^{22}$. 
En el Reino Unido durante 2009-10 un estimado de 572000 personas (2\% de la población laboral) reportó un desorden musculoesquelético que se creía originado o agravado por el trabajo ${ }^{23}$.

Similar a lo encontrado en este estudio, Zechinatti y colaboradores, a través del seguimiento a 10 años (1998-2008) de una cohorte de empleados de una Universidad estatal de Brasil, reportan que los desórdenes musculoesqueléticos y del tejido conectivo son unos de los diagnósticos responsables de la mayoría de las ausencias por enfermedad durante el periodo de estudio ${ }^{24}$.

La importancia de este estudio radica en el conocimiento del panorama del ausentismo laboral por causa médica en una empresa minera con el fin de orientar acciones de prevención de la enfermedad y promoción de la salud. Es necesario hacer seguimiento a los ausentistas repetitivos y crónicos para la identificación de posibles factores explicativos.

\section{REFERENCIAS BIBLIOGRÁFICAS}

1. Taylor P. Absenteeism, definition and statistics London, 1983. 8-16 p.

2. Hackett R, Guion R. A reevaluation of the absenteeism-job satisfaction relationship. Organizational Behavior and Human Decision Processes. 1985; 35: 340-81.

3. Nicholson N. Management Sanctions and Absence Controls. Human Relations. 1976; 29: $139-51$.

4. Rhodes S, Steers M. Managing employee absenteeism. Adisson-Wesley, editor. United States, 1990.

5. Koslowsky M, Sagie A, Kraus M, Dolam-Singer A Correlates of employee lateness: some theoretical considerations. Journal of Applied Psychology, 1997; 82: 79-88.

6. Steers R, Rhodes S. Major influences on employee atendance: A process model. Journal of Applied Psychology, 1978; 63: 391-407.

7. Steers R, Rhodes S. Knowledge and speculation about absenteeism. In: Jossey-Bass, editor. Absenteeism new approaches to understanding, measuring, and managin employee absence. San Francisco, 1984. p. $229-75$.

8. Iverson R, Olekalns M, Erwin P. Affectivity, Organizational stressors, and absenteeism: A causal model of burnout and its consequences. Journal of Vocational Behavior. 1998; 52: 1-23.

9. Iverson R, Deery S. Understanding the personological basis of employee withdrawal : The influence of affective disposition on employee tardiness, early departure, and absenteeism. Journal of Applied Psychology. 2001; 86 (5): 3-4.

10. Brayfield A, Crocket W. Employee attitudes and employee performance. Psychological Bulletin, 1955; 52 : 396-424.

11. Ortiz Y. Modelo de Steers y Rhodes sobre absentismo laboral. In: Unversidad de Granada, editor. Factores psicosociales del ausentismo laboral en la administración pública. Granada, 2003.

12. Mesa F, Kaempffer A. 30 años de estudio sobre ausentismo laboral en Chile: una perspeciva por tipos de empresa. Rev Méd Chil 2004; 132: 1100-8.

13. Cantuarias J, Cornejo E. Ausentismo laboral en una empresa minera: Tendencia 1985-1988. Rev Méd Chil 1993; $121: 827-36$.

14. Departamento Administrativo Nacional de Estadística. Producto Interno Bruto. Cuarto trimestre y total anual 2011. Santafé de Bogotá: 2012.

15. World Health Organisation. The ICD-10 Classification of Diseases, 1992.

16. ICONTEC. Norma Técnica Colombiana NTC 3793: Salud ocupacional. Clasificación, registro y estadística de ausentismo laboral. Santafé de Bogotá: ICONTEC; 1996, p. 2.

17. Bórquez P, Ruiz J. Ausentismo laboral: Análisis de las licencias médicas en un servicio médico de una empresa. Rev Méd Santiago, 1999; 2 (9): 15-8.

18. Danatro D. Ausentismo laboral de causa médica en una institución pública. Montevideo:1994-1995. Publicaciones del Sindicato Médico de Uruguay: Sindicato Médico de Uruguay, 1997.

19. Parada M, Moreno R, Rincón E, Mejía Z, Mora D, Rivas F. Ausentismo laboral de causa médica. Instituto Autónomo Hospital Universitario de los Andes. Mérida. Venezuela. 2001-2003. Revista de Facultad de Medicina, Universidad de Los Andes, 2006; 15: 22-6. 
20. Ferrie J, Vahtera J, Kivimäki M, Westerlund H, Melchior M, Alexanderson K, et al. Diagnosis-specific sickness absence and all-cause mortality in the GAZEL study. J Epidemiol Community Health. 2009; 63 (1): 50-5.

21. Gomero R, Llap C. Absentismo laboral de origen médico en el hospital Toquepala en el 2001. Rev Med Hered, 2004; 15 (2): 96-101.

22. Black C. Sickness absence and musculoskeletal disorders. What can be done? Rheumatology 2012; 51 : 204-5.

23. Health and safety executives. Musculoesqueletal disorders http://www.hse.gov.uk/statistics/lfs/swit3w12.xls 2011 [cited 2012 Julio].

24. Zechinatti C, Belloti J, Ynoe V, Albertoni W. Occupational musculoskeletal and mental disorders as the most frequent associations to worker's sickness absence: A 10-year cohort study. BMC Research Notes 2012; 5: 229.

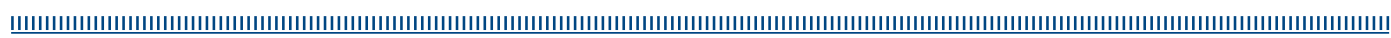

